\title{
An Heuristic Method for GPS Surveying Problem
}

\author{
Stefka Fidanova \\ IPP - Bulgarian Academy of Sciences, Acad. G. Bonchev str. bl.25A, 1113 Sofia, \\ Bulgaria \\ stefka@parallel.bas.bg
}

\begin{abstract}
This paper describes metaheuristic algorithm based on simulated annealing method,which is a nature-inspired method, to analyze and improve the efficiency of the design of Global Positioning System (GPS) surveying networks. Within the context of satellite surveying, a positioning network can be defined as a set of points which are coordinated by placing receivers on these point to determine sessions between them. The problem is to search for the best order in which these sessions can be observed to give the best possible schedule. The same problem arise in Mobile Phone surveying networks. Several case studies have been used to experimentally asses the performance of the proposed approach in terms of solution quality and computational effort.
\end{abstract}

\section{Introduction}

The continuing research on naturally occurring social systems offers the prospect of creating artificial systems that generate practical solutions to many Combinatorial Optimization Problems (COPs). Metaheuristic techniques have evolved rapidly in an attempt to find good solutions to these problems within a desired time frame 9. They attempt to solve complex optimization problems by incorporating processes which are observed at work in real life 24. The paper proposes simulated annealing algorithm which provide optimal or near optimal solution for large networks with an acceptable amount of computational effort.

The Global Positioning System is a satellite-based radio-navigation system that permits land, sea and airborne users to determine their three-dimensional position, velocity and time. This can be achieved 24 hours a day in all weather. In addition the satellite navigation system has an impact in all related fields in geoscience and engineering, in particular on surveying work in quickly and effectively determining locations and changes in locations network. The purpose of surveying is to determine the locations of points on the earth. Measuring tapes or chains require that the survey crew physically pass through all the intervening terrain to measure the distance between two points. Surveying methods have undergone a revolutionary change over that last few years with the deployment of the satellite navigation systems. The most widely known space systems are: the American Global Positioning System (GPS), the Russian GLObal Navigation Satellite System (GLONASS), and the forthcoming European Satellite 
Navigation System (GALILEO). In this paper, it is the use of GPS to establish surveying networks that is being investigated. GPS satellites continuously transmit electrical signals to the earth while orbiting the earth. A receiver, with unknown position on the earth, has to detect and convert the signals transmitted from all of the satellites into useful measurements. These measurements would allow a user to compute a three-dimensional coordinates position for the location of the receiver.

The rest of the paper is organized as follows. The general framework for GPS surveying network problem as a combinatorial optimization problem is described in Section 2. Then the search strategy of the simulated annealing is explained in Section 3. The metaheuristic algorithm applied to GPS surveying network is outlined and the general case of the problem is addressed, by presenting several case studies and the obtained numerical results in Section 4. The paper ends with a summary of the conclusions and directions for future research.

\section{Formulation of the GPS Surveying Network Problem}

A GPS network is distinctly different from a classical survey network in that no inter-visibility between stations is required. In GPS surveying, after defining the locations of the points for an area to be surveyed, GPS receivers will be used to map this area by creating a network of these coordinated points. These points, control stations within the context of surveying, are fixed on the ground and located by an experienced surveyor according to the nature of the land and the requirements of the survey [7. At least two receivers are required to simultaneously observe GPS satellites, for a fixed period of time, where each receiver is mounted on each station. The immediate outcome of the observation is a session between these two stations. After completing the first stage of sessions observation and defining the locations of the surveyed stations, the receivers are moved to other stations for similar tasks till the whole network is completely observed according to an observation schedule. The total cost of carrying out the above survey, which is computed upon the criteria to be minimized, represents the cost of moving receivers between stations. The problem is to search for the best order, with respect to the time, in which these sessions can be observed to give the cheapest schedule $\mathrm{V}$, i.e.:

$$
\text { Minimize: } C(V)=\sum_{p \in R} C\left(S_{p}\right)
$$

where: $C(V)$ is the total cost of a feasible schedule $V$;

$S_{p}$ is the route of the receiver $p$ in a schedule;

$R$ is the set of receivers $R=\{1, \ldots, r\}$;

\section{Simulated Annealing Method}

The Simulated Annealing (SA) is a generalization of an optimization method for examine the equations of state and frozen states of $n$-body systems. The 
original Metropolis scheme [8] was that an initial state of a thermodynamic system was chosen at energy $E$ and temperature $T$, holding $T$ constant the initial configuration is perturbed and the change of energy $d E$ is computed. The current state of the thermodynamic system is analogous to the current solution to the combinatorial problem, the energy equation for the thermodynamic system is analogous to the objective function and ground state is analogous to the global minimum. The key objective of this paper is to find an effective solution in a short period of time with close to least cost for a given GPS network using Simulated Annealing method.

$\mathrm{SA}$ is a heuristic method that has been implemented to obtain good solutions of an objective function defined on a number of discrete optimization problems. This method has proved to be a flexible local search method and can be successfully applied to the majority of real-life problems [1/3,5,6,10,12. The origin of the algorithm is in statistical mechanics. The fundamental idea is to allow moves resulting in solutions of worse quality than the current solution in order to escape from local minimum. The probability of doing such a move is declared during the search. The algorithm starts by generating an initial solution and by initializing the so-called temperature parameter $T$. The temperature is decreased during the search process, thus at the beginning of the search the probability of accepting uphill moves is high and it gradually decreases. This process is analogous to the annealing process of metals and glass, which assume a low energy configuration when cooled with an appropriate cooling schedule. Regarding the search process, this means that the algorithm is the result of two combined strategies:

- random walk;

- iterative improvement.

The first phase permits the exploration of the search space. The advantages of the algorithm are:

- Simulated Annealing is proved to converge to the optimal solution of the problem;

- An easy implementation of the algorithm makes it very easy to adapt a local search method to a simulated annealing algorithm.

In order to implement SA for GPS surveying problem a number of decisions and choices have to be made. Firstly, the problem specific choices, which determine the way in which the GPS surveying is modeled in order to fit into the SA framework. In other words, it involves the definition of the solution space $Q$ and neighborhood structure $I$, the form of the objective function $C(V)$ and the way in which a starting solution $V$ is obtained. Secondly, the generic choices which govern the working of the algorithm itself, are mainly concerned with the components of the cooling parameters: control parameter $T$ and its initial starting value, the cooling rate $F$ and the temperature update function, the number of iterations between decreases $L$ and the condition under which the system will be terminated 11. The performance of the achieved result is highly dependent on the right choice of both specific and generic desisions. 
The SA procedure used in this work is designed and developed essentially from practical experience and the requirement of the GPS surveying. A simple constructive procedure is proposed to obtain an initial (starting) feasible scheduling $V$ for the GPS surveying. The aim of this simple procedure is to obtain quickly an initial schedule. The structure of the SA algorithm is shown below:

1. The Problem Specific Decisions

Step 1. Formulation the problem parameters;

Step 2. Determination of the initial schedule, generate a feasible solution $V$;

2. The Problem Generic Decisions

Initialization the cooling parameters:

Set the initial starting value of the temperature parameter, $T>0$;

Step 3. Set the temperature length, $L$;

Set the cooling ratio, $F$;

Set the number of iterations, $K=0$.

3. The Generation Mechanism, Selecting and Acceptance Strategy of Generated Neighbors

Select a neighbor $V^{\prime}$ of $V$ where $V^{\prime} \in I(V)$

Step 4. Let $C\left(V^{\prime}\right)=$ the cost of the schedule $V^{\prime}$

Compute the move value $\Delta=C\left(V^{\prime}\right)-C(V)$

If $\Delta \leq 0$ accept $V^{\prime}$ as a new solution and set $V=V^{\prime}$

ELSE

Step 5. IF $e^{-\Delta / T}>\Theta$ set $V=V^{\prime}$, where $\Theta$ is a uniform random number $0<\Theta<1$

OTHERWISE retain the current solution $V$.

4. Updating the Temperature

Step 6. Updating the annealing schedule parameters using the cooling schedule $T_{k+1}=F * T_{k} k=\{1,2, \ldots\}$.

5. Termination of the Solution

Step 7. IF the stopping criteria is met THEN

Show the output

Step 8. Declare the best solution

OTHERWISE Go to step 4.

\section{Simulated Annealing Algorithm for GPS Surveying}

To understand the simulated annealing method, the Local Search (LS) strategy is throughly introduced. It is based on the concept of searching the local neighborhood of the current schedule [14. The LS procedure perturb a given solution to generate different neighborhoods using a move generation mechanism. In 
general, neighborhood for large-size problems can be much complicated to search. Therefor, LS attempts to improve an initial schedule $v$ to a GPS network by a series of local improvements. A move generation is a transition from a schedule $V$ to another one $V^{\prime} \in I(V)$ in one step (iteration). These schedules are selected and accepted according to some pre-defined criteria. The returned schedule $V$ may not be optimal, but it is the best schedule in its local neighborhood $I(V)$. A local optimal schedule is a schedule with the local minimal cost value. The basic steps for the LS procedure are as follows:

- Compute the cost value $C(V)$ of the current schedule $V$;

- Generate a schedule $V^{\prime} \in I(V)$ and compute its cost value $C\left(V^{\prime}\right)$;

- If $C(V)<C\left(V^{\prime}\right)$ then, $V^{\prime}$ replaces $V$ as a current schedule;

- Otherwise, retain $V$ and generate other moves until $C\left(V^{\prime}\right)<C(V)$ for all $V^{\prime} \in I(V)$;

- Terminate the search and return $V$ as the local optimal schedule.

The local search is an essential part of the simulated annealing method. In Saleh [13, a practical local search procedure that satisfies the GPS requirements has been developed. In this procedure, which is based on the sequential session interchange the potential pair-swaps are examined in order $(1,2),(1,3), \ldots,(1, n)$, $(2,3),(2,4), \ldots,(n-1, n), n$ is the number of sessions. The solution is represented by graph. The nodes correspond to the sessions and the edges correspond to the observation order.
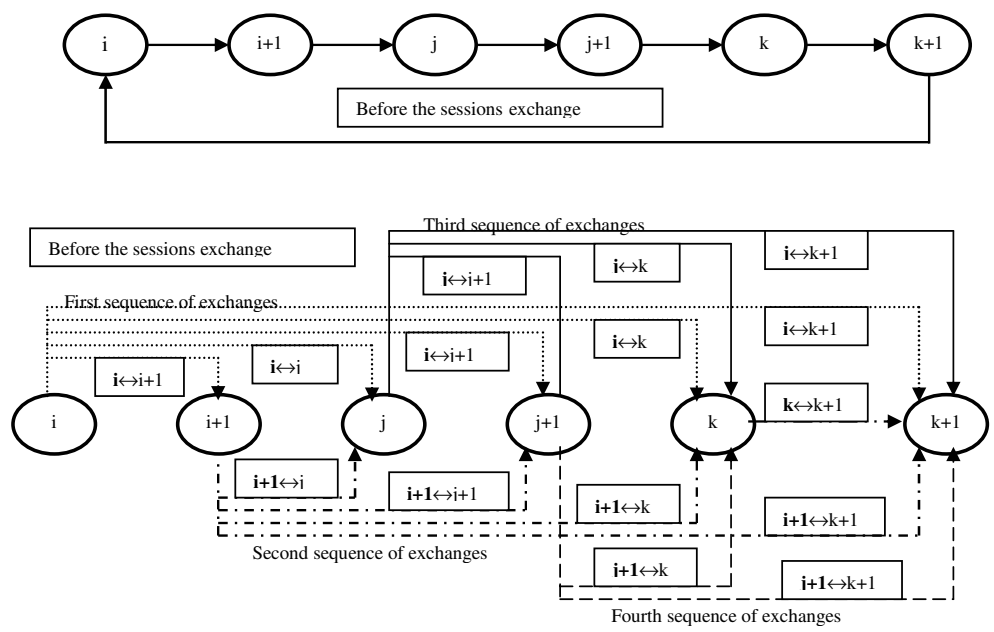

Fig. 1. The sequential local search procedure

In our algorithm we apply different local search procedure. We choose the potential pair-swaps randomly, in 13 they are chosen sequentially. When the best neighbor solution is equal to the global best solution, we choose randomly 
new current solution from the set of neighbors. The aim is to prevent $\Delta=0$ and thus to prevent repetition of the same solutions. It is a way for diversification of the search process.

\section{$5 \quad$ Experimental Results}

In this section some experimental results are reported. We compare simulated annealing algorithm used in 13 with our variant of the simulated annealing. Like a test problems we use real data of Malta and Seychelles GPS networks. The Malta GPS network is composed of 38 sessions and the Seychelles GPS network is composed of 71 sessions. We use 6 large test problems too from http: / / www.informatik.uni-heidelberg.de/groups / comopt/ software/ TSLIB95/ ATSP.html. These test problems consist from 100 to 443 sessions.

Table 1. Comparison of simulated annealing algorithms applied to various types of GPS networks

\begin{tabular}{|l|r|r|r|}
\hline Test & Nodes & sequential LS & random LS \\
\hline Malta & 38 & 1345 & 1035 \\
\hline Seychelles & 71 & 986 & 965 \\
\hline rro124 & 100 & 125606 & 59033 \\
\hline ftv170 & 170 & 6942 & 5179 \\
\hline rbg323 & 323 & 3787 & 1867 \\
\hline rbg358 & 358 & 2762 & 1749 \\
\hline rbg403 & 403 & 4852 & 2757 \\
\hline rbg443 & 443 & 6509 & 3111 \\
\hline
\end{tabular}

We use the following parameters for both algorithms: initial temperature is $20 \%$ of the result, final temperature is 3 , cooling parameter is 0.99 , number of iterations is 10 times the number of the nodes, number of swaps is $120 \%$ of the number of the nodes. The programs are written on $\mathrm{C}++$ language and are run on Pentium 4 PC with $2.8 \mathrm{GHz} 512$ RAM. The reported results are average over 25 runs of the algorithms.

Comparing the both simulated annealing algorithms, our algorithm outperforms other for all tested problems. In our algorithm we include random search and we prevent repeating of same solutions. It is a way for diversification of the search, to climb the hills and thus the algorithm has a greater possibility to find new and better solutions. 


\section{Conclusion}

In this paper a new simulated annealing algorithm for GPS surveying problem has been developed and compared with the algorithm used by Saleh in [13. A comparison of the performance of the both simulated annealing algorithms applied on various GPS networks is reported. The obtained results are encouraging and the ability of the developed techniques to generate rapidly high-quality solutions for observing GPS networks can be seen. The problem is important because it arises in wireless communications like GPS and mobile phone communications and can improve the services in the networks. Thus the problem has an economical importance. For future work we will investigate the influence of the parameter settings and their dependence of the number of the nodes and the expected results.

Acknowledgments. The author is supported by European community grant BIS $21++$.

\section{References}

1. Arts E., Van Loarhoven P.: Statistical Cooling: A General Approach to Combinatorial Optimization Problems, Phillips Journal of Research 40, (1985), 193-226

2. Bonabeau E., Dorigo M., Theraulaz G.: Swarm Intelligence: From Natural to Artificial Systems, Oxford University Press, New York.(1999)

3. Cerny V.: A Thermodynamical Approach to the Traveling Salesman Problem: An Efficient Simulated Annealing Algorithm, J. of Optimization Theory and Applications 45, (1985), 41-51

4. Corne D., Dorigo M., Glover F. (eds.): New Ideas in Optimization, McCraw Hill, London.(1999)

5. Dowsland K.: Variants of Simulated Annealing for Practical Problem Solving In Rayward-Smith V. ed., Applications of Modern Heuristic Methods, Henley-onThames: Alfred Walter Ltd. in association with UNICOM, (1995)

6. Kirkpatrick S., Gelatt C.D., Vecchi P.M.: Optimization by Simulated Annealing, Science 220, (1983),671-680

7. Leick, A.: GPS Satellite Surveying, 2nd. edition. Wiley, Chichester, England.(1995)

8. Metropolis N., Rosenbluth A., Rosenbluth M., Teller A., Teller E.: Equation of State Calculations by Fast Computing Machines, J. of Chem Phys. 21(6), (1953), 1087-1092

9. Osman I.H., Kelly J.P.: Metaheuristics:An overview. In Osman I.H., Kelly J.P. eds. Metaheuristics:Theory and Applications, Kluwer Acad. Publisher.(1996)

10. Osman I.H., Potts C.N.: Simulated Annealing for Permutation Flow-Shop Scheduling, Omega 17, (1989), 551-557

11. Reeves C. R. (ed.): Modern Heuristic Techniques for Combinatorial Problems, Oxford, England, Blackwell Scientific Publications, (1993)

12. Rene V.V.: Applied Simulated Annealing, Berlin, Springer, (1993)

13. Saleh H. A., and Dare P.: Effective Heuristics for the GPS Survey Network of Malta: Simulated Annealing and Tabu Search Techniques. Journal of Heuristics N 7 (6), (2001), 533-549

14. Schaffer A. A., Yannakakis M.: Simple Local Search Problems that are Hard to Solve, Society for Industrial Applied Mathematics Journal on Computing, Vol 20, (1991), pp. 56-87 